D. MINDA

KODAI MATH. J.

8 (1985), 249-258

\title{
ESTIMATES FOR THE HYPERBOLIC METRIC
}

\author{
BBy David Minda
}

\begin{abstract}
Bounds for the density of the hyperbolic metric of a hyperbolic region $X$ in the complex plane $\boldsymbol{C}$ or on the Riemann sphere $\boldsymbol{P}$ are given in terms of the euclidean or spherical distance to the boundary of $X$. Also, bounds for the infimum of the density of the hyperbolic metric are given in terms of the supremum of the radii of all disks in $X$. These bounds are related to various Landau constants and are implicit in previous work on finding lower bounds for Landau constants.
\end{abstract}

1. Introduction. Let $X$ denote a hyperbolic region in the complex plane $\boldsymbol{C}$; that is, $\boldsymbol{C} \backslash X$ contains at least two points. The hyperbolic, or Poincaré, metric on $X$ is denoted by $\lambda_{X}(z)|d z|$. It is a complete Riemannian metric on $X$ with constant curvature -4 . Recall that

$$
\lambda_{D}(z)|d z|=\frac{|d z|}{1-|z|^{2}},
$$

where $\boldsymbol{D}=\{z:|z|<1\}$ is the unit disk. Typically, there is no explicit formula for the density $\lambda_{X}(z)$ of the hyperbolic metric, so estimates are useful. However, there are few results that deal explicity with the size of the hyperbolic metric. Let us survey some of these. Ahlfors ([1], [2]) gave analytic bounds in case $X$ is the thrice punctured sphere. Often, one is interested in bounds for $\lambda_{X}(z)$ in terms of the geometric quantity $\delta_{X}(z)$ which is the enclidean distance from $z$ to the boundary of $X$. The upper bound $\lambda_{X}(z) \leqq 1 / \delta_{X}(z)$ is a direct consequence of Schwarz' lemma [6, p. 45]. If $X$ is simply connected, then $\lambda_{X}(z) \geqq 1 / 4 \delta_{X}(z) \quad[6$, p. 45]. This lower bound is equivalent to the Koebe one-quarter theorem. If $X$ is convex, then the factor 4 in the lower bound can be replaced by 2 [9]. Blevins [4] obtained a sharp lower bound for simply connected regions that are bounded by a quasiconformal circle. Beardon and Pommerenke ([3], [12]) investigated bounds in terms of $\delta_{X}(z)$ and another geometric quantity. In particular, they determined a necessary and sufficient condition on a region $X$ for the existence of a positive constant $c$ such that $\lambda_{X}(z) \geqq c / \delta_{X}(z)$. The condition is that there exists a positive constant $M$ such that the modulus of any annulus in $X$ that separates $\partial X \cup\{\infty\}$ is at most $M$. Hence, it is necessary that $\partial X$ have no isolated points.

We are interested in obtaining a lower bound for $\lambda_{X}(z)$ in terms of $\delta_{X}(z)$ that is valid even if the boundary of $X$ has isolated points. The clue to the

Received June 29, 1984 
form of the bound is provided by examining the hyperbolic metric of a punctured disk. If $X=\{z: 0<|z-a|<R\}$, then

$$
\lambda_{X}(z)=\frac{1}{2|z-a| \log (R /|z-a|)} .
$$

Then $\delta_{X}(z)=|z-a|$ for $0<|z-a| \leqq R / 2$ and so

$$
\lambda_{X}(z)=\frac{1}{2 \delta_{X}(z) \log \left(R / \delta_{X}(z)\right)}
$$

for $z$ in $X$ neara. This example also shows explicitly that $\lambda_{X}(z) \delta_{X}(z)$ has no positive lower bound as $z$ approaches $a$. It also suggests that for a general hyperbolic region we consider the possibility of finding a lower bound of the form

$$
\lambda_{X}(z) \geqq \frac{1}{2 \delta_{X}(z) \log \left(b / \delta_{X}(z)\right)},
$$

where $b$ is a positive constant. Such a bound is implicit in Ahlfors' method for determining a lower bound for the Landau constant [1]. This idea is also used in [8]. 'Since bounds for the hyperbolic metric are relatively scarce, it seems worthwhile to make explicit these bounds.

Let $\Delta(X)$ be the supremum of $\delta_{X}(z)$ as $z$ ranges over $X$. We only consider lower bounds of the form (1) with $b \geqq \Delta(X)$; this insures that the lower bound is positive in $X$. Since the right-hand side of (1) is a decreasing function of $b$ on the interval $(\Delta(X), \infty)$, we let $b(X)$ denote the infimum of all constants $b>$ $\Delta(X)$ such that (1) holds for all $z \in X$ in order to obtain the best possible lower bound of the form (1) when $b$ is replaced by $b(X)$. Set $b(X)=\infty$ if there is no lower bound of the form (1). We shall show that $e^{1 / 2} \Delta(X) \leqq b(X) \leqq e \Delta(X)$. Consequently, there is a lower bound of the form (1) for the density of the hyperbolic metric lif and only if there is a uniform bound on the size of the disks that are contained in $X$. As an application of this result we show that $\lambda_{X}(z)$ has a positive lower bound if and only if $\Delta(X)$ is finite. More precisely, if $\Lambda(X)=\inf \left\{\lambda_{X}(z): z \in X\right\}$, then $1 / 2 \leqq \Delta(X) \Lambda(X) \leqq 1$. The upper bound is sharp, but the lower bound $1 / 2$ is not. The best possible lower bound is related to Landau's constant. For convex regions we show that $\pi / 4$ is the sharp lower bound.

Finally, we consider analogs of the preceding results for regions on the Riemann sphere $\boldsymbol{P}$. In this context we consider the "spherical" density $(1+$ $\left.|z|^{2}\right) \lambda_{X}(z)$ which is invariant under rotations of $\boldsymbol{P}$ and seek estimates of this quantity in terms of the size of the largest spherical disk in $X$ with center $z$.

2. Lower bound for plane regions. In this section we consider lower bounds for the density of the hyperbolic metric in a plane region.

THEOREM 1. Let $X$ be a hyperbolic region in the complex plane $C$. Then $e^{1 / 2} \Delta(X) \leqq b(X) \leqq e \Delta(X)$. 
Proof. We first establish the lower bound for $b(X)$. Of course, there is no harm in assuming that $b(X)<\infty$. Then (1) holds for $b=b(X)$. Since the upper bound $\lambda_{X}(z) \leqq 1 / \delta_{X}(z)$ holds in any hyperbolic region in $C$, we obtain from (1)

or

$$
\frac{1}{2 \log \left(b(X) / \delta_{X}(z)\right)} \leqq 1
$$

$$
e^{1 / 2} \delta_{X}(z) \leqq b(X)
$$

This yields $e^{1 / 2} \Delta(X) \leqq b(X)$.

Next, we demonstrate the upper bound for $b(X)$ under the assumption that $\Delta=\Delta(X)<\infty$. We begin by assuming that we actually have the strict inequality $\delta_{X}(z)<\Delta$ for all $z \in X$. Define

$$
\rho(z)|d z|=\frac{|d z|}{2 \delta_{X}(z) \log \left(e \Delta / \delta_{X}(z)\right)} .
$$

We will show that $\rho(z)|d z|$ is an ultrahyperbolic metric on $X$. The inequality $\rho(z) \leqq \lambda_{X}(z)$ will then follow from Ahlfors' generalization of Schwarz' lemma ([1], [2, p. 13]). Since $\delta_{X}(z)$ is a continuous function, it is clear that $\rho(z)|d z|$ is a positive continuous metric on $X$. To show that $\rho(z)|d z|$ is an ultrahyperbolic metric on $X$, we must exhibit a supporting metric at each point $z_{0}$ of $X$. This is a metric $\lambda_{0}(z)|d z|$ defined in a neighborhood of $z_{0}$ with constant curvature -4 such that $\lambda_{0}(z) \leqq \rho(z)$ for $z$ near $z_{0}$ with equality at $z_{0}$. Given $z_{0} \in X$, select $a \in \partial X$ with $\left|z_{0}-a\right|=\delta_{X}\left(z_{0}\right)$. Then $\delta_{X}(z) \leqq|z-a|<\Delta$ for $z$ near $z_{0}$ with equality at $z_{0}$. The hyperbolic metric for $\{z: 0<|z-a|<e \Delta\}$ is

$$
\lambda_{0}(z)|d z|=\frac{|d z|}{2|z-a| \log (e \Delta /|z-a|)} .
$$

Because the function $h(t)=1 / 2 t \log (e \Delta / t)$ is decreasing on $(0, \Delta]$ and increasing on $[\Delta, e \Delta)$, the inequality $\delta_{X}(z) \leqq|z-a|<\Delta$ for $z$ near $z_{0}$ yields $\lambda_{0}(z) \leqq \rho(z)$ for $z$ close to $z_{0}$ with equality at $z_{0}$. Since the hyperbolic metric $\lambda_{0}(z)|d z|$ has constant curvature -4 , it is a supporting metric for $\rho(z)|d z|$ at $z_{0}$. Then Ahlfors' lemma yields (1) with $b=e \Delta$. Hence, $b(X) \leqq e \Delta$ in case $\delta_{X}(z)<\Delta$ for all $z \in X$. In the general case, just replace $\Delta$ by $\Delta_{n}=\Delta+(1 / n), n$ any positive integer, and obtain $b(X) \leqq e \Delta_{n}$. Finally, let $n$ tend to infinity to conclude that $b(X) \leqq e \Delta$ in the general case.

As an application of Theorem 1 we obtain estimates for $\Lambda(X)$, the infimum of the hyperbolic metric in $X$, in terms of the quantity $\Delta(X)$.

THEOREM 2. Let $X$ be a hyperbolic region in $C$. Then

$$
\frac{1}{2 \Delta(X)} \leqq \Lambda(X) \leqq \frac{1}{\Delta(X)} \text {. }
$$

Proof. We begin by proving the upper bound. Since $\lambda_{X}(z) \leqq 1 / \delta_{X}(z)$ for any hyperbolic plane region with equality at $z$ if and only if $X$ is a disk with center 
$z[6$, p. 45], the upper bound is immediate. Also $\Lambda(X)=1 / \Delta(X)$ for any disk.

Second, we establish the lower bound. If $\Delta=\Delta(X)=\infty$, then there is nothing to prove, so we may assume $\Delta<\infty$. Then from Theorem 1

$$
\lambda_{X}(z) \geqq \frac{1}{2 \delta_{X}(z) \log \left(e \Delta / \delta_{X}(z)\right)} .
$$

Since $h(t)=1 / 2 t \log (e \Delta / t)$ has its minimum value $1 / 2 \Delta$ on the interval $(0, e \Delta)$ at the point $t=\Delta$, we have $\lambda_{X}(z)>1 / 2 \Delta$. Then $\Lambda(X) \geqq 1 / 2 \Delta$.

The best possible constant $C$ such that $\Lambda(X) \geqq C / \Delta(X)$ for any hyperbolic region $X$ in $\boldsymbol{C}$ is related to Landau's constant $\mathcal{L}$. We briefly recall the definition of $\mathcal{L}$. In our notation $\mathcal{L}=\inf \Delta(f(\boldsymbol{D}))$, where the infimum is taken over all holomorphic functions $f$ defined in $\boldsymbol{D}$ and normalized by $f^{\prime}(0)=1$. Assume that $f$ is such a function, $X=f(\boldsymbol{D})$ and $\Delta(X)<\infty$. Then for $w \in X$

$$
\frac{C}{\Delta(X)} \leqq \Lambda(X) \leqq \lambda_{X}(w) \text {. }
$$

The principle of hyperbolic metric $[5$, p. 336] yields

so that

$$
\lambda_{X}(f(z))\left|f^{\prime}(z)\right| \leqq \lambda_{D}(z)=\frac{1}{1-|z|^{2}},
$$

$$
\frac{C}{\Delta(X)} \leqq \lambda_{X}(f(0)) \leqq 1 .
$$

Consequently, $C \leqq \Delta(X)$ and so $C \leq \mathcal{L}$. From Theorem 2 we obtain the known lower bound $\mathcal{L} \geqq 1 / 2$ that is due to Ahlfors [1]. This is not surprising since the lower bound in Theorem 1 is implicit in [1]. The best known upper bound for the Landau constant is [13]

$$
\mathcal{L} \leqq \frac{\Gamma(1 / 3) \Gamma(5 / 6)}{\Gamma(1 / 6)}<.5433
$$

and this bound is conjectured to be the actual value of the Landau constant. It seems plausible that $C=\mathcal{L}$. We now demonstrate that the analogous result is valid for convex regions.

TheORem 3. Let $X$ be a convex hyperbolic region in $C$. Then

$$
\frac{\pi}{4 \Delta(X)} \leqq \Lambda(X) \leqq \frac{1}{\Delta(X)}
$$

and both bounds are sharp.

Proof. In the proof of Theorem 2 we already noted that the upper bound is sharp for any disk. Now, we establish the lower bound and its sharpness. Let $X$ be any convex hyperbolic region in $C$ with $\Delta=\Delta(X)<\infty$. Then Minda [9] obtained the lower bound 


$$
\lambda_{X}(z) \geqq \frac{\pi}{4 \Delta \sin \left(\frac{\pi \delta_{X}(z)}{2 \Delta}\right)} \geqq \frac{\pi}{4 \Delta} .
$$

(Actually, in [9] the denominator in the lower bound has the factor 2 rather than 4. This is due to the fact that the hyperbolic metric was normalized to have curvature -1 rather than -4 in [9].) Thus, $\Lambda(X) \geqq \pi / 4 \Delta$. Finally, we demonstrate the sharpness. If $S=\{z: 0<\operatorname{Re}(z)<2 M\}$, then

$$
\lambda_{S}(z)=\frac{\pi}{4 M \sin \left(\frac{\pi \operatorname{Re}(z)}{2 M}\right)} \geqq \frac{\pi}{4 M}=\frac{\pi}{4 \Delta(S)}
$$

with equality for $\operatorname{Re}(z)=M$. Thus, $\Lambda(S)=\pi / 4 \Delta(S)$. Actually, equality holds for any strip.

Recall that the Bloch-Landau constant for convex regions is $\pi / 4$ ([9], [14]).

3. Lower bounds for spherical regions. For a plane region $X$ the density of the hyperbolic metric can be viewed as the quotient of the hyperbolic metric $\lambda_{X}(z)|d z|$ and the euclidean metric $|d z|$. Note that $\lambda_{X}(z)$ is invariant under translations and rotations of $\boldsymbol{C}$. For a region on the Riemann sphere $\boldsymbol{P}$ we need to determine the proper analog of the density. Throughout the remainder of this section we assume that $X$ denotes a hyperbolic region on $\boldsymbol{P}$. The spherical metric $|d z| /\left(1+|z|^{2}\right)$ is a Riemannian metric on $\boldsymbol{P}$ with constant curvature 4. This metric is invariant under the group of rotations of the sphere. Precisely, if either $T(z)=e^{i \theta}(z-a) /(1+\bar{a} z), \quad a \in C$, or else $T(z)=e^{i \theta} / z$, where $\theta \in \boldsymbol{R}$, then

$$
\frac{\left|T^{\prime}(z)\right|}{1+|T(z)|^{2}}=\frac{1}{1+|z|^{2}}
$$

We define the spherical hyperbolic density of the hyperbolic metric to be the quotient of the hyperbolic metric and the spherical metric; in symbols,

$$
\mu_{X}(z)=\frac{\lambda_{X}(z)|d z|}{\frac{|d z|}{1+|z|^{2}}} .
$$

If $T$ is a rotation of the sphere, then the conformal invariance of the hyperbolic metric [9] yields

It follows that

$$
\lambda_{T(X)}(T(z))\left|T^{\prime}(z)\right|=\lambda_{X}(z)
$$

$$
\mu_{T(X)}(T(z))=\mu_{X}(z),
$$

so the spherical hyperbolic density is invariant under rotations of the sphere. Observe that if $0 \in X$, then $\mu_{X}(0)=\lambda_{X}(0)$. Set $M(X)=\inf \left\{\mu_{X}(z): z \in X\right\}$.

Next, we need a notion of distance on the sphere. Define 


$$
d(z, w)= \begin{cases}\left|\frac{z-w}{1+\bar{w} z}\right| & \text { if } \quad z, w \in \boldsymbol{C}, \\ \frac{1}{|z|} & \text { if } \quad z \in \boldsymbol{C}, w=\infty .\end{cases}
$$

The quantity $d(z, w)$ is invariant under all rotations $T$ of $\boldsymbol{P}$; that is, $d(T(z)$, $T(w))=d(z, w)$, but it is not a true distance function. However, it is related to the chordal distance $\chi$ and the spherical distance $\phi$ by

$$
\begin{gathered}
\chi(z, w)=\frac{|z-w|}{\sqrt{1+|z|^{2}} \sqrt{1+|w|^{2}}}=\sin \left(\tan ^{-1}(d(z, w))\right), \\
\phi(z, w)=\tan ^{-1}(d(z, w)) .
\end{gathered}
$$

Recall that $\psi(z, w)$ denotes half the angle at the center of the sphere subtended by the shorter arc of the great circle connecting $z$ and $w$. Because of this connection with $\chi(z, w)$ and $\phi(z, w)$ we shall employ $d(z, w)$ as a measure of distance on $\boldsymbol{P}$. The advantage of this approach is a simplicity in the formulas and a clearer analogy with the results in the planar case. Of course, all results could be expressed in terms of $\chi$ or $\psi$ instead of $d$ by making use of their relationship. For $a \in \boldsymbol{P}$ and $r>0$ let $D(a, r)=\{z \in \boldsymbol{P}: d(a, z)<r\}$. This is a spherical disk with center $a$ and radius $r$. The boundary of $D(a, r)$ is a euclidean circle when we view $D(a, r)$ on the Riemann sphere. For $r=1$ we obtain a hemisphere with center $a$. For $z \in X$ let $\varepsilon_{X}(z)$ denote the largest value of $r$ such that $D(z, r) \subset X$. The geometric quantity $\varepsilon_{X}(z)$ is a measure of the spherical distance from $z$ to $\partial X$. We are interested in estimating $\mu_{X}(z)$ in terms of $\varepsilon_{X}(z)$ and $M(X)$ by means of $E(X)=\sup \left\{\varepsilon_{X}(z): z \in X\right\}$.

THEOREM 4. Let $X$ be a hyperbolic region on the Riemann sphere $\boldsymbol{P}$. Then $\mu_{X}(z) \leqq 1 / \varepsilon_{X}(z)$ for $z \in X$. If equality holds at a point $z$, then $X$ is a spherical disk with center $z$.

Proof. Because of the rotational invariance of the quantities $\mu_{X}(z)$ and $\varepsilon_{X}(z)$, there is no harm in assuming that $z=0$. In this case $\mu_{X}(0)=\lambda_{X}(0)$ and $\varepsilon_{X}(0)=$ $\delta_{X}(0)$, so the conclusion $\mu_{X}(0) \leqq 1 / \varepsilon_{X}(0)$ is equivalent to the known bound $\lambda_{X}(0) \leqq$ $1 / \delta_{X}(0)[6, \mathrm{p} .45]$. If equality holds in this latter inequality, then $X$ is a disk centered at the origin. In the general case $X$ would be a rotation of a disk centered at the origin; that is, $X$ would be a spherical disk.

This theorem helps to show that the geometric quantity $\varepsilon_{X}(z)$ is a reasonable candidate for estimating $\mu_{X}(z)$. The following example will motivate the form of our lower bound for $\mu_{X}(z)$.

EXAMPLE 1. Let us calculate $\mu_{X}(z)$ for a punctured spherical disk. Let $X=$ $\{z: 0<d(a, z)<R\}$. Let $T$ be a rotation of $\boldsymbol{P}$ that sends $a$ to the origin. Then $T(X)=\{z: 0<|z|<R\}$ and 


$$
\begin{aligned}
\mu_{X}(z) & =\mu_{T(X)}(T(z))=\left(1+|T(z)|^{2}\right) \lambda_{T(X)}(T(z)) \\
& =\frac{1+|T(z)|^{2}}{2|T(z)| \log (R /|T(z)|)} \\
& =\frac{1+d^{2}(a, z)}{2 d(a, z) \log (R / d(a, z))},
\end{aligned}
$$

since $d(a, z)=d(T(a), T(z))=d(0, T(z))=|T z|$. For $d(a, z)$ small we have $d(a, z)$ $=\varepsilon_{X}(z)$ and so

$$
\mu_{X}(z)=\frac{1+\varepsilon_{X}^{2}(z)}{2 \varepsilon_{X}(z) \log \left(R / \varepsilon_{X}(z)\right)} .
$$

In view of the preceding example we seek a lower bound of the form

$$
\mu_{X}(z) \geqq \frac{1+\varepsilon_{X}^{2}(z)}{2 \varepsilon_{X}(z) \log \left(c / \varepsilon_{X}(z)\right)},
$$

where $c \geqq E(X)$ is a positive constant. Let $c(X)$ be the smallest such constant. We wish to estimate $c(X)$ in terms of $E(X)$. Since $C \backslash\{0\}$ is not hyperbolic and $E(\boldsymbol{C} \backslash\{0\})=1$ because $\boldsymbol{C} \backslash\{0\}$ contains a hemisphere but no larger spherical disk, it is plausible that a restriction $E(X)<1$ be imposed in order to obtain a bound of the form (2).

THEOREM 5. Let $X$ be a hyperbolic region on $\boldsymbol{P}$. Then $E(X) \exp \left(\left(1+E^{2}(X)\right)\right.$ $/ 2) \leqq c(X)$. If $E(X)<1$, then $c(X) \leqq E(X) \exp \left(\left(1+E^{2}(X)\right) / 2\left(1-E^{2}(X)\right)\right)$.

Proof. We start by establishing the lower bound for $c(X)$ under the assumption that $c(X)<\infty$. Then (2) holds with $c=c(X)$. Because $\mu_{X}(z) \leqq 1 / \varepsilon_{X}(z)$, we obtain from $(2)$

or

$$
\frac{1+\varepsilon_{X}^{2}(z)}{2 \log \left(c(X) / \varepsilon_{X}(z)\right)} \leqq 1
$$

$$
\varepsilon_{X}(z) \exp \left(\left(1+\varepsilon_{X}^{2}(z)\right) / 2\right) \leqq c(X) .
$$

The lower bound follows immediately.

Now we derive the upper bound under the assumption that $E=E(X)<1$. Initially, we assume that $\varepsilon_{X}(z)<E$ for all $z \in X$. We shall show that

$$
\rho(z)|d z|=\frac{1+\varepsilon_{X}^{2}(z)}{2 \varepsilon_{X}(z) \log \left(A / \varepsilon_{X}(z)\right)} \frac{|d z|}{1+|z|^{2}}
$$

is an ultrahyperbolic metric on $X$, where $A=E \exp \left(\left(1+E^{2}\right) / 2\left(1-E^{2}\right)\right)$. Fix $z_{0} \in X$; we will construct a supporting metric at $z_{0}$. Select $a \in \partial X$ with $\varepsilon_{X}\left(z_{0}\right)=d\left(a, z_{0}\right)$. Then $\varepsilon_{X}(z) \leqq d(a, z)<E$ for $z$ near $z_{0}$ with equality at $z_{0}$. From Example 1 it follows that the hyperbolic metric on the punctured disk $\{z: 0<d(a, z)<A\}$ is

$$
\lambda_{0}(z)|d z|=\frac{1+d^{2}(a, z)}{2 d(a, z) \log (A / d(a, z))} \frac{|d z|}{1+|z|^{2}} .
$$


The fact that the function $k(t)=\left(1+t^{2}\right) / 2 t \log (A / t)$ is decreasing on $(0, E]$ and increasing on $[E, A)$ together with the inequality $\varepsilon_{X}(z) \leqq d(a, z)<E$ for $z$ near $z_{0}$ imply that $\lambda_{0}(z)|d z| \leqq \rho(z)|d z|$ for $z$ near $z_{0}$ with equality at $z_{0}$. Thus $\lambda_{0}(z)|d z|$ is a supporting metric for $\rho(z)|d z|$ at $z_{0}$. Ahlfors' lemma yields $\lambda_{X}(z)|d z| \geqq \rho(z)|d z|$. If we divide both sides of this inequality by the spherical metric $|d z| /\left(1+|z|^{2}\right)$, then we obtain (2) with $c=A$. Hence, $c(X) \leqq A$ in case $\varepsilon_{X}(z)<E$ for all $z \in X$. In the general case, replace $E$ by $E_{n}=E+(1 / n)$, where the positive integer $n$ is taken so large that $E_{n}<1$. Then obtain $c(X) \leqq A_{n}$. Let $n$ tend to infinity to get $c(X) \leqq A$ in the general case.

Theorem 6. Let $X$ be a hyperbolic region on $\boldsymbol{P}$. Then

$$
\frac{1}{E(X)}-E(X) \leqq M(X) \leqq \frac{1}{E(X)} \text {. }
$$

Proof. The upper bound follows easily from Theorem 4. Equality holds for any spherical disk. The lower bound is nonpositive for $E(X) \geqq 1$, so we may assume that $E=E(X)<1$ in the course of establishing it. Then Theorem 5 gives

$$
\mu_{X}(z) \geqq \frac{1+\varepsilon_{X}^{2}(z)}{2 \varepsilon_{X}(z) \log \left(A / \varepsilon_{X}(z)\right)},
$$

where $A=E \exp \left(\left(1+E^{2}\right) / 2\left(1-E^{2}\right)\right)$. Since $k(t)=\left(1+t^{2}\right) / 2 t \log (A / t)$ attains its minimum value $E^{-1}-E$ on the interval $(0, A)$ at the point $t=E$, we have $\mu_{X}(z)$ $\geqq E^{-1}-E$. Hence, $M(X) \geqq E^{-1}-E$.

The lower bound for $M(X)$ in terms of $E(X)$ is not sharp. The best possible lower bound is related to Landau constants for meromorphic functions; for more information about these constants the reader is directed to [8].

EXAMPLE 2. For each positive integer $n$ let $X_{n}$ denote the complex plane punctured at both the origin and all the $n^{t h}$ roots of unity. For instance, $X_{1}$ is the Riemann sphere punctured at 0,1 and $\infty$. We wish to determine a lower bound for $\lambda_{n}(z)$, the hyperbolic density on $X_{n}$. Theorem 1 is of no help in this situation because $X_{n}$ contains arbitrarily large euclidean disks. We shall make use of Theorem 6 . Set $E_{n}=E\left(X_{n}\right)$. Triviallv, $E_{1}=1$. Elementary geometric considerations show that for $n \geqq 2 \quad E_{n}=(\sqrt{3+\cos (2 \pi / n)}-\sqrt{1+\cos (2 \pi / n)}) / \sqrt{2}$. In particular, $E_{2}=1, E_{3}=(\sqrt{5}-1) / \sqrt{2}$ and $E_{4}=(\sqrt{3}-1) / \sqrt{2}$. We see that $E_{n}<1$ only for $n \geqq 3$ so we can apply Theorem 6 to obtain a meaningful lower bound in these cases. For $n \geqq 3$ we obtain

$$
\lambda_{n}(z) \geqq\left(\frac{1}{E_{n}}-E_{n}\right) /\left(1+|z|^{2}\right) .
$$

As special cases we have

$$
\lambda_{3}(z) \geqq \frac{1}{1+|z|^{2}},
$$




$$
\lambda_{4}(z) \geqq \frac{\sqrt{2}}{1+|z|^{2}} .
$$

For $n \geqq 3$ we have shown that the hyperbolic metric on $X_{n}$ dominates a constant multiple of the spherical metric.

It is possible to obtain similar lower bounds for $\lambda_{1}(z)$ and $\lambda_{2}(z)$ by making use of the following device. The function $p(z)=z^{4}$ is a covering of $X_{4}$ onto $X_{1}$ so the invariance of the hyperbolic metric under a covering [7] yields

$$
\lambda_{4}(z)=\lambda_{1}(p(z))\left|p^{\prime}(z)\right|=\lambda_{1}\left(z^{4}\right) 4|z|^{3} .
$$

If we set $w=z^{4}$, then

$$
\begin{aligned}
\lambda_{1}(w) & =\lambda_{4}(z) / 4|z|^{3} \\
& \geqq \frac{1}{2 \sqrt{2}} \frac{1}{1+|w|^{2}} \frac{1+|z|^{8}}{|z|^{3}+|z|^{5}} \\
& \geqq \frac{1}{2 \sqrt{2}} \frac{1}{1+|w|^{2}} .
\end{aligned}
$$

This lower bound is implicit in the work of Pommerenke [11]. By making use of the covering $q(z)=z^{2}$ of $X_{2}$ onto $X_{1}$ one can demonstrate in a similar manner that

$$
\lambda_{2}(z) \geqq \frac{1}{\sqrt{2}} \frac{1}{1+|z|^{2}} .
$$

Thus, in all cases $\lambda_{n}(z)$ dominates an explicit scalar multiple of $1 /\left(1+|z|^{2}\right)$.

\section{REFERENCES}

[1] Ahlfors, L. V., An extension of Schwarz's lemma, Trans. Amer. Math. Soc., 43 (1938), 359-364.

[2] Ahlfors, L.V., Conformal invariants. Topics in geometric function theory, McGraw-Hill, New York, 1973.

[3] Beardon, A.F. and Ch. Pommerenke, The Poincaré metric of plane domains, J. London Math. Soc. (2) 18 (1978), 475-481.

[4] Blevins, D.K., Conformal mappings of domains bounded by quasi-conformal circles, Duke Math. J., 40 (1973), 877-883.

[5] Goluzin, G.M., Geometric theory of functions of a complex variable, Translations of Mathematical Monographs, 26, Amer. Math. Soc., Providence, 1969.

[6] KRA, I., Automorphic functions and Kleinian groups, W. A. Benjamin, Reading, Mass., 1972.

[7] Minda, C.D., The hyperbolic metric and coverings of Riemann surfaces, Pacific J. Math., 84 (1979), 171-182.

[8] Minda, C.D., Bloch constants, J. Analyse Math. 41 (1982), 54-84.

[9] Minda, C.D., Lower bounds for the hyperbolic metric in convex regions, Rocky Mtn. J. Math., 13 (1983), 61-69.

[10] OsGOOD, B., Some properties of $f^{\prime \prime} / f^{\prime}$ and the Poincaré metric, Indiana Univ. 
Math. J., 31 (1982), 449-461.

[11] Pommerenke, CH., Normal functions, Proc. NRL Conf. on Classical Function Theory, Math. Res. Center, Naval Res. Lab., Washington, D. C., 1970, pp. 77-93.

[12] Pommerenke, $C_{\text {H. }}$, Uniformly perfect sets and the Poincaré metric, Arch. Math., 32 (1979), 192-199.

[13] Rademacher, H., On the Bloch-Landau constant, Amer. J. Math., 65 (1943), 387-390.

[14] SzEGö, G., Über eine Extremalaufgabe aus der Theorie der schlichten Abbildungen, Sitzungsberichte der Berliner Mathematische Gesellschaft, 22 (1923), 38-47.

Department of Mathematical Sciences

UNIVERSITY OF CinCINNATI

Cincinnati, Ohio 45221

U.S.A. 